\title{
How to Customize Architecture under Heavily Prescribed Design Conditions? Principles and Prospects for an Evolutionary System
}

\author{
Victor Bunster \\ University of Melbourne, Australia \\ v.bunster@student.unimelb.edu.au
}

\begin{abstract}
Architectural design is a complex activity. The development of a building involves management of the diverse goals of a heterogeneous group of agents using no more than the discrete resources of a given setting. These variables can often conflict and result in rigid normative frameworks that can limit the capacity of a designer to respond with accuracy to diverse environmental factors. The main aim of this paper is to present the theoretical foundations of an evolutionary system to assist the customization of architecture under such prescribed design conditions.
\end{abstract}

Keywords: Mass customization; Design computing; Information theory; Evolution; Prescription.

\section{Introduction}

Our built environments show a persistent tendency towards repetition. If something characterizes current cities it might be the ubiquitous presence of highly redundant patterns at different scales of buildings and neighborhoods. Some of the reasons behind this phenomenon might be clear to some extent; however, avoiding the architectural problems that may result from this way of building is not a minor issue.

The industrial revolution had a significant impact on architecture. From being a craft largely defined by manual labor, the design of buildings became increasingly dominated by the possibilities of serially manufactured products. As early noted by Mumford (1930) this change brought contrasting consequences to architecture. The economies of scale of assembly lines lowered construction costs, opening new target markets and technical possibilities for buildings. However, this move towards standardization also implied stronger constraints being imposed on the work of designers. Mass production rests on the economic advantages of large numbers of repeated units, a logic that tends towards homogeneity and limits the capacity of designers to respond with accuracy to the diverse variables that characterize their environment.

In this context, mass customization emerges as a paradigm-shift for industrial production, aiming towards the individualization of serially-manufactured units to meet the heterogeneity and dynamism of consumers' expectations (Pine II, 1993). Mass customization enables flexibility in serial production through effective management of higher amounts of information content. As explained by Mitchell (2009[2003]), computer-aided design and manufacturing (CAD/CAM) techniques result in design worlds that may not be conceivable without computers. These enhanced possibilities may inform buildings able to respond with higher accuracy to complex environmental conditions (Mitchell 2004; 2005). However, these open possibilities are still far from mainstream, where the logic of mass customization may reach its full potential.

This paper presents the theoretical foundations of a computerbased system to assist architectural customization in contexts that are traditionally far from technological advances, targeting a relatively unexplored area of design computing: the problem of fostering variability in near deterministic design settings.

\section{Constraints and Design Spaces}

Among the differences between design and open speculation may be the constraints that put limits to what can be considered a potentially satisfactory outcome. A designer is expected to respond to specific needs of the diverse agents involved in the development of a building using no more than the means available for that given situation. These interacting variables define a design space that sets the boundaries for any speculative explorations. This conceptual tool may be used to explain why in architecture a combination of conflicting requirements and limited resources can often result in redundant typologies.

A number of authors have recognized the significant role that constraints play in the act of designing. Simon (1996[1972]), for example, explains design as a problem similar to optimization in which a number of constraints and requirements need to be satisficed. Similarly, Lawson (1990[1980]) identifies the origins of design problems in different types of constraint generators that would frame the work of designers. Gedenryd (1998) differentiates between rigid, somewhat flexible, and completely flexible constraints according to their level of prescription. While rigid constraints generally come from legislators as mandatory 
requirements, user-specific needs and client expectations can be somewhat flexible as they are generally open to personal interpretation. In contrast, designer-imposed constraints are optional and completely flexible. A designer may decide to explore the possibilities of e.g. a determinate style, material, or geometry, but may also dispose of them discretionally without a straightforward effect on the quality of the outcomes.

Despite being optional, designer-imposed constraints play a significant role during the form definition process. As explained by Simon (1996[1972]), our cognitive capabilities prevent us to follow truly optimizing behavior. Decision-makers act according to their own capabilities, using only the information that is available in a discrete time framework to settle in a solution that may only meet the acceptability threshold. Such limitations shall also apply to designers, as the complexity of a design space may exceed their capacity to understand and process information from the environment. Consequently, self-imposed constraints help practitioners to explore discrete parts of a design space with enough depth to refine adequate outcomes (Figure 1) (Gedenryd, 1998).

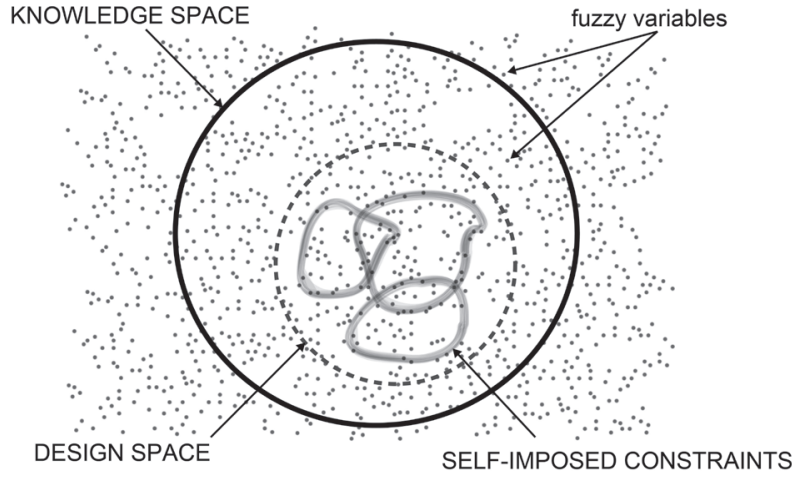

Figure 1: Schematic representation of a design space

Self-imposed constraints are open-ended and therefore enable substantial differentiation of designs coming from an otherwise homogeneous setting. Different designers may approach the open areas of a design space using different strategies that may result in contrasting solutions. This may explain why interaction of conflicting interests often results in design spaces with a strong tendency towards repetition. The development of a building is an expensive and time-consuming process in which the goals and expectations of diverse groups often collide. This is the case of some massive real estate projects and public housing developments (Figure 2). Political, social and economic concerns can conflict and impose rigid normative requirements to designers, reducing the open areas that enable speculative explorations. A common outcome of excessive prescription is buildings that respond only to the uttermost rigid of their mandatory regulations and fail to meet the heterogeneous needs of their users and context.

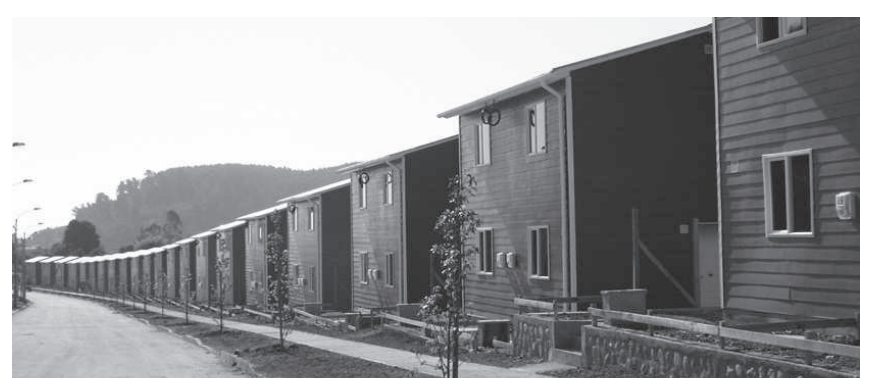

Figure 2: Highly repetitive housing typology as a result of a heavily prescribed design space (source: Author)

Nevertheless, the level of prescription of a design space may also be linked to the means available in each situation. When the intricacy of a design space overcomes the capabilities of a particular designer, a natural response might be to focus only on the conditions that ensure minimum fitness of the outcomes. This implies that a system able to enhance the capabilities of designers when managing environmental conditions may result in expanded design options.

\section{Modularity and Customization}

For Mitchell (2004; 2005), the current proliferation of intricate building morphologies such as the Bilbao Guggenheim and Boston's Stata Center may be a manifestation of our increasing ability to manage information. The shift from the sketching board to CAD/CAM systems would imply more than a simple change of medium. Digital technologies enable management of amounts of information beyond the capabilities of any designer, enhancing the potential variability of the outcomes.

Despite of these open possibilities, mass customization is still scarce in mainstream practice. This may be a consequence of conditions that are specific to the building industry, including limited availability of advanced CAD/CAM systems, the high construction costs and slow obsolescence of buildings, or even plain resistance to change among different agents. Nevertheless, it may be possible to increase the variability of buildings within the means of mainstream practice. Mitchell $(2004 ; 2005)$ explains that buildings such as Habitat 67 or the Nakagin Capsule Tower achieve high levels of complexity by exploiting the combinatorial possibilities of repeated modules. This implies that it may be possible to increase the potential variability of a design space whilst avoiding prohibitive construction costs or the need of advanced fabrication machinery (Figure 3 ).

These principles have an analogous in the design and manufacturing industry. Significant advances on mass customization have focused on the possibilities of modular product families composed by discrete modules to be assembled as different versions of a same product. This strategy enables ondemand alteration of the quantities produced to meet the fluctuating requirements of the market. 

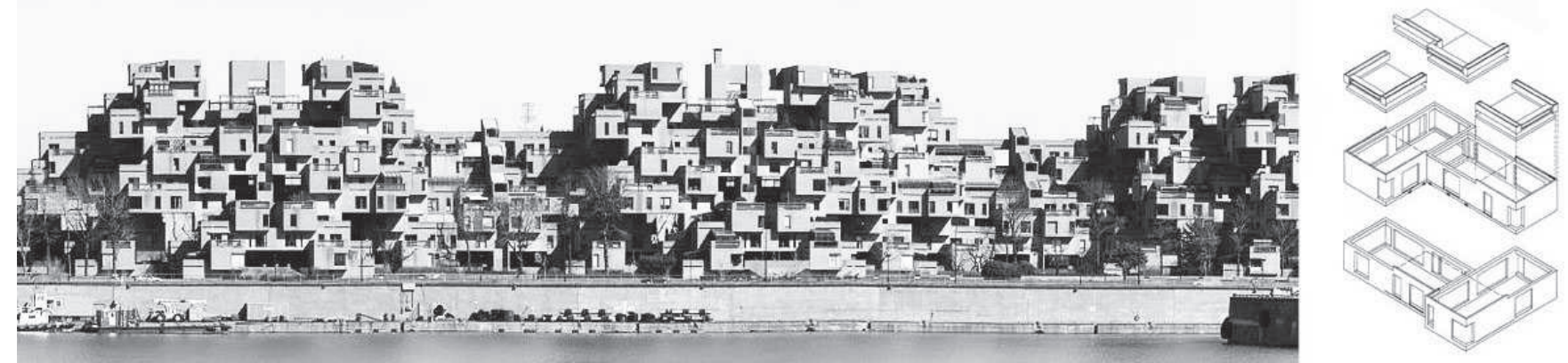

Figure 3: Complex design morphology using repetitive construction in Safdie's Habitat 67 (source: C.A.C.-McGill University)

In traditional mass production any change requires loss of time and major infrastructural transformations, thus becoming inefficient. Modular product families enable cost-optimization by incorporating such changes beforehand, enabling flexibility that may result in increased long-term profits (Jiao \& Tseng, 1999).

Gero and Sosa (2008) explain that this approach to mass customization can be considered as routine design (Gero, 1990) as it enables diverse outcomes within a well-defined design space. This entails potential variability in an otherwise repetitive framework, hence a feasible strategy to increase its scope within prescriptive limitations. Nevertheless, the definition of a modular product family able to respond to a wide range of environmental variables using only a discrete set of modules is a significant problem. This issue may be tackled with evolution of self-imposed constraints using complexity maximization as search criteria.

\section{Design Complexity and Information}

From an information processing perspective, the immediate result of the design activity is a design description that joins the decisions and knowledge of a designer with a materialized building (Gero, 1990). As explained by Mitchell (2004; 2005), this description may take the form of e.g. a one-dimensional string of text, a twodimensional drawing, a three-dimensional model, or a multidimensional database. Different mediums differ significantly in their capabilities for managing information, hence may result in differences in the potential complexity of a building.

Mitchell $(2004 ; 2005)$ explains that the information content of a design description may be directly linked to its capacity to respond with accuracy to the environment. Accordingly, he defines design complexity (DC) as the ratio of added design content (ADC) to added construction content (ACC):

$$
D C=A D C / A C C
$$

Where $A D C$ is the information needed for the specification of a building, and ACC is the resulting information for its construction. Under this framework, a short design description that produces high amount construction content shall result in a design of low complexity. This is the case of repetitive construction of traditional mass production methods. In contrast, non-repetitive buildings would need high amounts of information for their full specification, resulting in designs of high complexity. Interestingly, buildings such as Habitat 67 would achieve high levels of DC through ADC that results in a proportionally low ACC. Such strategy may be used to expand design options in mainstream practice.

Mitchell (2004: 1473) suggests the use of information theory as a framework to quantify the information content of a design description. Similarly, Gero and Sosa (2008) explain that Shannon's entropy can be used to measure the morphological complexity design prototypes. In this case, Shannon's entropy is used to quantify the $D C$ of a modular product family that acts as routine design space.

Information theory works with an idealized model of communication. In simple terms, this model consists of an information source that uses a transmitter to encode a message, which is sent through a channel to a destination that decodes it with a receiver. The success of this information transmission depends upon the bandwidth of the channel and the possibility of errors represented by a noise source. If a designer is seen as the source of information, the message can be analogous to a design description that is received by a construction agent (Figure 4).

This information theoretical framework enables quantification of the potential complexity of a design description using Shannon's entropy (Gero \& Sosa, 2008). Entropy is a probabilistic measurement of the average information content of a message. Given a random stream of binary digits, for example, its entropy is the amount of useful information it can potentially encrypt. In other words, entropy is the average unpredictability of a random variable, which is equivalent to its potential information content and therefore to its potential complexity. Accordingly, anything that is not entropic is considered redundant and can be removed without any loss of information (see source code theorem in Shannon, 1948):

$$
\text { entropy }=\text { message }- \text { redundancy }
$$




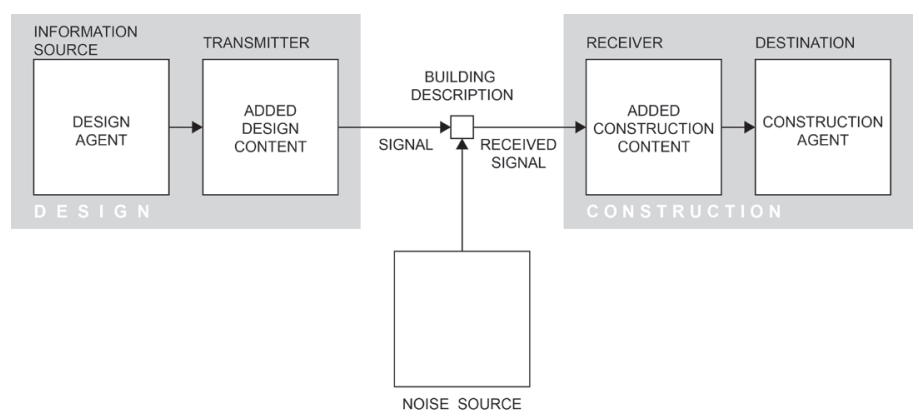

Figure 4: Information theoretical model of design when embedded in a CAD/CAM system (adapted from Shannon, 1948)

In design, redundancy may be analogous to repetition: the more repetitive a design is, the lower its potential complexity. Then, a design description with a significant tendency towards repetition should have low entropy and scarce space for customization. Mitchell's DC can be quantified using Shannon's entropy under this framework, enabling measurement of the potential customization of a design description when encrypted as digital information.

\section{Design as Constraint Evolution}

Genetic algorithms (GA) are among the most versatile techniques available for the resolution of complex optimization problems (Bentley, 1999). In architecture, this capacity has been extensively used in the definition of designs that respond efficiently to diverse environmental constraints (Caldas, 2001; Sharples, Holden \& Pasquarelli, 2002). These properties make GA a suitable strategy to pursue complexity in modular design spaces.

Gero and Sosa (2008) propose an evolutionary system that targets novelty using Shannon's entropy as complexity maximization criteria. They demonstrate that this strategy can be used to expand design options within the limited means of industrial production. A similar strategy may use Mitchell's DC as search criteria to generate modular product families of enhanced variability (Figure 5).

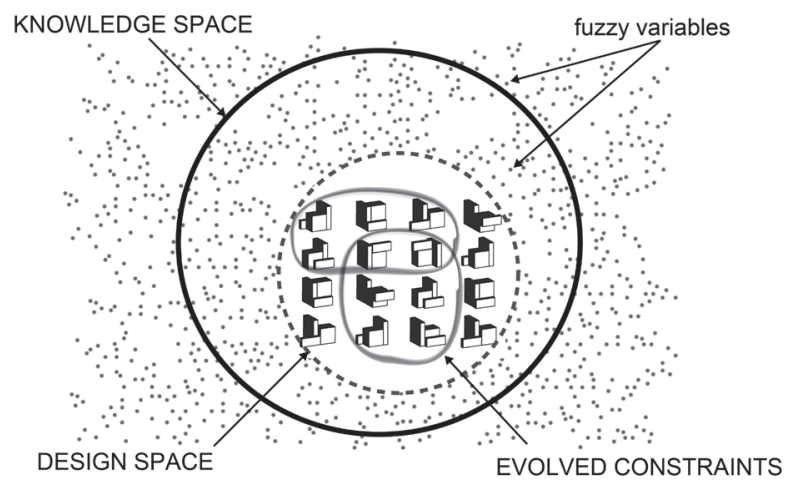

The proposed evolutionary system works at three complementary steps: the generation of i) modular building structures, the selection of ii) discrete product families, and the specification of iii) adequate configurations according to location- and userspecific requirements.

\section{Modular structures}

This first step consists in the definition of a set of modular building components and their potential combinations. A GA that uses DC as selection criteria is used to generate a range of modular structures that respond optimally to the uttermost rigid of the constraints that define a design space (Figure 6-left). The result of this first step is an expansion of the potential design options within prescriptive limitations.

\section{Discrete product families}

The second step consists in the selection of a discrete product family among the evolved modular building components. Using DC as selection criteria, a GA identifies the combination of modules that may offer higher combinatorial possibilities and therefore can result in enhanced variability (Figure 6-middle). This search enables definition of discrete but flexible modular architectures that enable accurate responses to environmental constraints whilst maintaining affordability.

\section{Context-specific configurations}

The third step consists in the specification of a design solution using the evolved modular product family as routine design space. The heterogeneity of this product family shall enables customization of outputs using resources similar to those of mass production. At this stage, a GA is used to find the combinatorial structure that responds with higher accuracy to given environmental conditions (Figure 6-right).

Figure 5: Schematic representation of a modular design space 


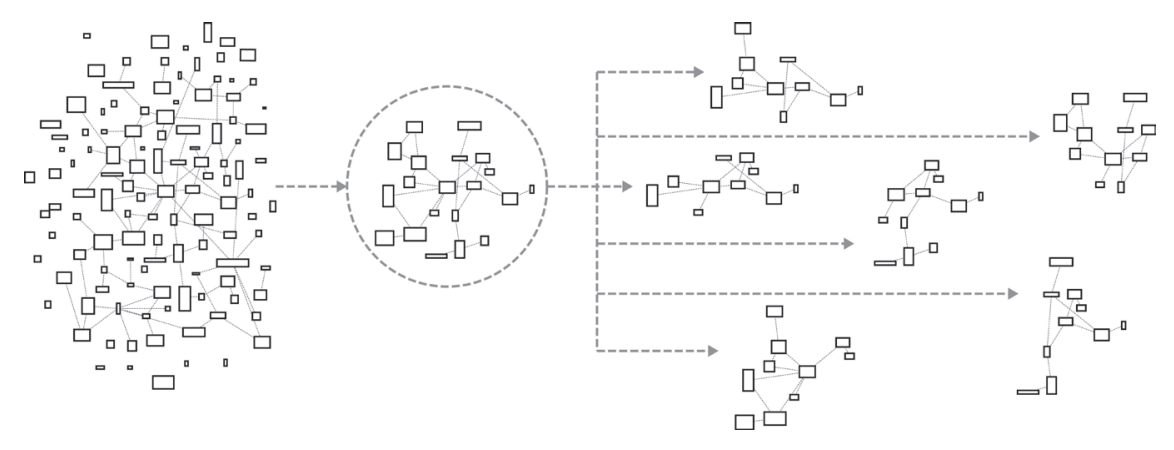

Figure 6: Stages of the proposed evolutionary system: (left) and increase of the potential complexity of a design space with the generation of modular building components and relational structures, (middle) management of this complexity with the selection of a discrete product family, and (right) definition of optimal configurations according to specific environmental conditions

\section{Discussion and Further Development}

This paper discusses the theoretical foundations of an evolutionary system to assist designers in the problem of customizing architecture under heavily prescribed design conditions. The notion of design space is used to explain how interaction of conflicting constraints may result in rigid normative frameworks, and how the means available for the development of a design description may affect the capacity for outcomes to respond to complex environmental conditions. This conceptual standpoint suggests information theory and Shannon's entropy are feasible frameworks to formalize a design description and quantify its potential complexity when embedded in a CAD/CAM system. These principles inform an evolutionary system that relies on evolution of design constraints to pursue flexibility within rigid normative frameworks and on modular building families to ensure their compliance with prescriptive standards.

Further work will focus on the development of the proposed evolutionary system as a software tool, and on its implementation in a case studio to explore its possibilities and limitations in an existing normative framework.

\section{Acknowledgments}

The work presented in this paper is part of a Ph.D. research currently under development at C.R.I.D.A. - Faculty of Architecture, Building and Planning of The University of Melbourne, with the financial support of CONICYT - Chile. The author thanks Professor Bharat Dave, Dr. Stanislav Roudavski, and Edan Weis for their advice and comments throughout the development of this paper.

\section{References}

Bentley, P. J. (1999). An introduction to evolutionary design by computers. In P.J. Bentley (Ed.), Evolutionary Design by Computers (pp. 1-73). San Francisco, CA: Morgan Kaufmann Publishers.
Caldas, L. G. (2001). An evolution-based generative design system: Using adaptation to shape architectural form (Doctoral dissertation). MIT, Cambridge, MA.

Duarte, J. P. (2005). Towards the mass customization of housing: The grammar of Siza's houses at Malagueira. Environment and Planning B, 32(3), 347-380.

Gero, J. S. (1990). Design prototypes: A knowledge representation schema for design. Al Magazine, 11(4), 26-36.

Gero, J. S., \& Sosa, R. (2008). Complexity measures as a basis for mass customization of novel designs. Environment and Planning B, 35(1), 3-15.

Jiao, J., \& Tseng, M. M. (1999). A methodology of developing product family architecture for mass customization. Journal of Intelligent Manufacturing, 10(1), 3-20.

Mitchell, W. J. (2009[2003]). Design worlds and fabrication machines. In B. Kolarevic (Ed.), Architecture in the digital age: Design and manufacturing (pp. 107-114). New York: Spon Press.

Mitchell, W. J. (2004). Beyond the ivory tower: Constructing complexity in the digital age. Science, 303, 1472-1473.

Mitchell, W. J. (2005). Constructing complexity. In B. Martens \& A. Brown (Eds.), Proceeding of the Tenth International Conference on Computer Aided Architectural Design Futures (pp. 41-50). Vienna: Springer.

Mumford, L. (1930). Mass production and the modern house, Part I. The Architectural Record, 67, 13-20.

Pine II, B. J. (1993). Mass customization: The new frontier in business competition. Boston, MA: Harvard Business School Press.

Shannon, C. E. (1948). A mathematical theory of communication. Bell System Technical Journal, 27(3), 379-423.

Sharples, C., Holden, K., \& Pasquarelli, G. (Eds.). (2002). Versioning: Evolutionary techniques in architecture. London: Academy Editions.

Simon, J. H. (1996[1969]). The sciences of the artificial; 3rd edition. Cambridge MA: The MIT Press. 